\title{
On the use of the Choquet integral with fuzzy numbers in multiple criteria decision support
}

\author{
Patrick Meyer $^{\mathrm{a}, *}$, Marc Roubens ${ }^{\mathrm{b}}$ \\ a Service de Mathématiques Appliquées, Université du Luxembourg, 162a, avenue de la Faïencerie, L-1511 Luxembourg \\ ${ }^{b}$ Mathématique \& Recherche Opérationnelle, Faculté Polytechnique de Mons, 9, rue de Houdain, B-7000 Mons, Belgium
}

\begin{abstract}
This paper presents a multiple criteria decision support approach in order to build a ranking and suggest a best choice ${ }^{1}$ on a set of alternatives. The partial evaluations of the alternatives on the points of view can be fuzzy numbers. The aggregation is performed through the use of a fuzzy extension of the Choquet integral. We detail how to assess the coefficients of the aggregation operator by using alternatives which are well-known to the decision maker, and which originate from his domain of expertise.
\end{abstract}

Keywords: Multiple criteria decision support; Fuzzy partial evaluations; Choquet integral; Choice; Ranking

\section{Introduction}

This paper presents a multiple criteria decision support approach which aims at building a ranking and a best choice recommendation on a set of alternatives $A$. The work is based on a multiple criteria sorting procedure proposed in [12] and later in [13]. Here, we present an extension to the general case where alternatives are described by fuzzy partial evaluations. In real life problems, this extension can be very useful, as very often it is difficult to determine an exact value for alternatives on certain points of view.

As an example one can consider the hypothetical problem of the localisation of a large factory in a country. The alternatives represent different areas and among the points of view one can find for example the number of direct and indirect working places which will be created. It seems obvious that for most of the areas it will be hard to determine their exact evaluation on this point of view. Nevertheless, it is probably possible to determine a range of values which may apply with more or less credibility. This small example shows that the use of imprecise information as evaluations can be useful in a lot of situations.

One possible way to represent such imprecise information is by fuzzy numbers. In particular, in the context of multiple criteria decision support, it can be quite useful to consider trapezoidal or triangular fuzzy numbers (see Section 2) for the partial evaluations.

\footnotetext{
* Corresponding author. Tel.: +35 24666446673 .

E-mail address: patrick.meyer@uni.lu (P. Meyer).

${ }^{1} \mathrm{~A}$ best choice is a subset of alternatives which can be considered as the best ones.
} 
We briefly recall general results in Section 2 and focus on trapezoidal and triangular fuzzy numbers. Then, in Section 3, we present the context of the multiple criteria decision support problem we are dealing with here. Section 4 recalls a few results on the aggregation by means of a Choquet integral. Later, in Section 5 we show how to extend the Choquet integral [4] by Zadeh's extension principle [20]. In Section 6, we present 2 possible rankings and a best choice procedure on the alternatives of $A$. An important point is dealt with in Section 7 where we show how to assess the coefficients of the Choquet integral from well-known alternatives.

\section{Preliminary considerations}

In this section, we first recall general concepts on fuzzy sets, fuzzy numbers and possibility distributions. In 1965 , Zadeh [20] introduced the concept of fuzzy set to be able to represent and manipulate data which have nonstatistical uncertainty or in case of impreciseness. Let $X$ be a classical set. A fuzzy set $\widetilde{Y}$ in $X$ can be defined by its membership function

$$
\mu_{\tilde{Y}}: X \rightarrow[0,1] .
$$

For $x \in X, \mu_{\widetilde{Y}}(x)=0$ means that $x$ does not belong to $\widetilde{Y}, \mu_{\widetilde{Y}}(x)=1$ represents the complete membership of $x$ to $\widetilde{Y}$, and the values between 0 and 1 stand for intermediate memberships. We write $\widetilde{Y}(x)=\mu_{\widetilde{Y}}(x)$ for the degree of membership of the element $x$ in the fuzzy set $\widetilde{Y}$, for each $x$ in $X$.

The support $\underline{\widetilde{Y}}$ of a fuzzy set $\widetilde{Y}$ of $X$ is the crisp set of elements of $X$ for which the membership degree to $\widetilde{Y}$ is non-zero,

$$
\underline{\tilde{Y}}=\{x \in X: \tilde{Y}(x)>0\} .
$$

A fuzzy set $\widetilde{Y}$ of $X$ is said to be normal if there exists an element $x$ in $\widetilde{Y}$ for which $\tilde{Y}(x)=1$. Let us call $\operatorname{cl}(\underline{\tilde{Y}})$ the closure of the support of $\widetilde{Y}$. A $\lambda$-level set of a fuzzy set $\tilde{Y}$ of $X$ is given by

$$
[\tilde{Y}]^{\lambda}= \begin{cases}\{x \in X: \tilde{Y}(x) \geqslant \lambda\} & \text { if } \lambda>0, \\ \operatorname{cl}(\underline{\tilde{Y}}) & \text { if } \lambda=0 .\end{cases}
$$

A fuzzy set $\widetilde{Y}$ of $X$ is said to be convex if $[\tilde{Y}]^{\lambda}$ is a convex subset of $\widetilde{Y}$ for all $\lambda \in[0,1]$.

A fuzzy number $\tilde{x}$ of $\mathbb{R}$ is a fuzzy set $\widetilde{Y}$ in $\mathbb{R}$ that is normal, convex and has a continuous membership function of bounded support. Let $\mathcal{F}$ be the family of all fuzzy numbers. For a fuzzy number $\tilde{x} \in \mathcal{F}$ we define

$$
a_{m}(\lambda)=\min [\widetilde{x}]^{\lambda}, \quad a_{M}(\lambda)=\max [\widetilde{x}]^{\lambda} .
$$

We now can introduce the concept of possibility distributions by means of the fuzzy numbers as defined in [20] and [21]. This allows to see fuzzy numbers as possibility distributions. Let $a, b \in \mathbb{R} \cup\{-\infty,+\infty\}$ with $a \leqslant b$. The possibility that $\tilde{x} \in \mathcal{F}$ takes its value from the interval $[a, b]$ is defined by

$$
\operatorname{Pos}(\tilde{x} \in[a, b])=\max _{x \in[a, b]} \tilde{x}(x) .
$$

In particular for $\lambda \in[0,1]$,

$$
\begin{aligned}
& \operatorname{Pos}\left(\tilde{x} \leqslant a_{m}(\lambda)\right)=\max _{x \leqslant a_{m}(\lambda)} \tilde{x}(x)=\lambda, \\
& \operatorname{Pos}\left(\tilde{x} \geqslant a_{M}(\lambda)\right)=\max _{x \geqslant a_{M}(\lambda)} \tilde{x}(x)=\lambda .
\end{aligned}
$$

In [5] the authors write a fuzzy number in a very general way as

$$
\tilde{x}(x)= \begin{cases}L\left(\frac{a-x}{\alpha}\right) & \text { if } a-\alpha \leqslant x \leqslant a, \\ 1 & \text { if } a<x \leqslant b, \\ R\left(\frac{x-b}{\beta}\right) & \text { if } b<x \leqslant b+\beta, \\ 0 & \text { otherwise, }\end{cases}
$$


where $[a, b]$ is the peak of $\tilde{x}, L, R:[0,1] \rightarrow[0,1]$ are upper semi-continuous and non-increasing shape functions with $L(0)=R(0)=1$ and $L(1)=R(1)=0$ which are called side functions. The support $\underline{\tilde{x}}$ is equal to $] a-\alpha, b+\beta[$. The crisp numbers are particular cases of the fuzzy numbers. If $\tilde{x} \in \mathcal{F}$ with $\underline{\tilde{x}}=\{a\}$ then $\tilde{x}$ is a crisp number.

A particular type of fuzzy numbers are the trapezoidal fuzzy numbers. They can be very useful in the framework of multiple criteria decision support which is presented in Section 3. A fuzzy number $\tilde{x}$ is called a trapezoidal fuzzy number if its membership function can be written as

$$
\tilde{x}(x)= \begin{cases}1-\frac{a-x}{\alpha} & \text { if } a-\alpha \leqslant x \leqslant a, \\ 1 & \text { if } a<x \leqslant b, \\ 1-\frac{x-b}{\beta} & \text { if } b<x \leqslant b+\beta, \\ 0 & \text { otherwise. }\end{cases}
$$

$\alpha$ and $\beta$ are called the left and right width. We use the notation $\tilde{x}=(a, b, \alpha, \beta)$. One can show that $[\tilde{x}]^{\lambda}=[a-(1-$ $\lambda) \alpha, b+(1-\lambda) \beta]$, for all $\lambda \in[0,1]$. In the particular case where $a=b$ the fuzzy number is called triangular and $a$ is said to be the centre of $\tilde{x}$.

In order to be able to use mathematical operations on the fuzzy numbers, Zadeh [20] introduces the sup-min extension principle which allows to work consistently with the crisp case. If $\widetilde{x_{1}}, \ldots, \widetilde{x_{n}} \in \mathcal{F}$ and $f: \mathbb{R}^{n} \rightarrow \mathbb{R}$ is a continuous function, then the sup-min extension principle is defined by

$$
f\left(\widetilde{x_{1}}, \ldots, \widetilde{x_{n}}\right)(y)=\sup _{f\left(x_{1}, \ldots, x_{n}\right)=y} \min \left\{\tilde{x_{1}}\left(x_{1}\right), \ldots, \tilde{x_{n}}\left(x_{n}\right)\right\} \quad \forall y \in \mathbb{R} .
$$

It has been shown in [14] that $f\left(\tilde{x_{1}}, \ldots, \tilde{x_{n}}\right)$ is a fuzzy number.

In the following section, we present the multiple criteria decision support problem which we aim at solving in this paper.

\section{A particular multiple criteria decision support framework}

Let $A$ be a set of $q$ alternatives and $N=\{1, \ldots, n\}$ be a label set of points of view. The partial evaluation of each alternative for each point of view is made on an interval scale. It accepts as admissible transformation function a positive linear transformation (see [16]). Moreover the evaluations are supposed to be commensurable. We can then suppose without any loss of precision that the performance scale of each point of view is the closed continuous unit interval $[0,1]$.

The particular case we are actually presenting here is where the evaluations of the alternatives on the points of view are fuzzy numbers. This means that each alternative $x \in A$ can be identified with its corresponding fuzzy profile

$$
x \equiv\left(\widetilde{x_{1}}, \ldots, \widetilde{x_{n}}\right) \in[0,1]^{n},
$$

where for any $i \in N, \tilde{x_{i}}$ represents the partial evaluation of $x$ on point of view $i$.

In order to suggest a ranking of the alternatives of $A$ to the decision maker, one possible way is given below:

- Aggregate the partial evaluations of each alternative on the points of view in a global evaluation.

- Order these global evaluations to obtain a ranking.

Classically, the weighted sum is often used as an aggregation operator. This additive representation, however, implies preferential independence of the points of view. One way to avoid this independence condition is to use the Choquet integral $[4,9]$ as an aggregation operator.

A further hypothesis has to be made here in order to be able to use Zadeh's extension principle. Let $\widetilde{x_{1}}, \ldots, \widetilde{x_{n}}$ be fuzzy numbers. A fuzzy number $\tilde{c}$ of $\mathbb{R}^{n}$ is called the joint possibility distribution of $\widetilde{x_{1}}, \ldots, \widetilde{x_{n}}$ if it satisfies

$$
\widetilde{c}\left(x_{1}, \ldots, x_{n}\right) \leqslant \min \left\{\widetilde{x_{1}}\left(x_{1}\right), \ldots, \tilde{x_{n}}\left(x_{n}\right)\right\} \quad \forall x_{i} \in \mathbb{R}, \quad i=1, \ldots, n .
$$


Equivalently $[\widetilde{c}]^{\gamma} \subseteq\left[\tilde{x}_{1}\right]^{\gamma} \times \cdots \times\left[\tilde{x}_{n}\right]^{\gamma}$ for all $\gamma \in[0,1]$. Fuzzy numbers $\widetilde{x_{i}} \in \mathcal{F}, i=1, \ldots, n$ are said to be noninteractive if $[\widetilde{c}]^{\gamma}=\left[\widetilde{x_{1}}\right]^{\gamma} \times \cdots \times\left[\widetilde{x_{n}}\right]^{\gamma}$ for all $\gamma \in[0,1]$. Zadeh implicitly supposes that the fuzzy numbers have to be non-interactive in the extension principle.

In the sequel, we suppose that the partial evaluations of the alternatives, represented by fuzzy numbers, are noninteractive (in the sense discussed above). Nevertheless, this does not mean that the points of view cannot interact. On the level of the points of view, different types of interactions can occur. For example, one can have substitution ${ }^{2}$, complementarity ${ }^{3}$ or preferential dependence ${ }^{4}$. These types of interactions among the points of view can be expressed by a Choquet integral (and clearly not by a weighted sum).

In the following section, we present this aggregation operator which allows to deal with interacting points of view, namely the Choquet integral.

\section{The Choquet integral as an aggregation operator}

Let us consider an alternative $x \in A$ which is described by crisp partial evaluations $x_{i},(i \in N)$. The Choquet integral of $x$ is then defined by

$$
\mathcal{C}_{v}(x):=\sum_{i=1}^{n} x_{(i)}\left[v\left(A_{(i)}\right)-v\left(A_{(i+1)}\right)\right],
$$

where $v$ represents a fuzzy measure on $N$, that is a monotone set function $v: 2^{N} \rightarrow[0,1]$ fulfilling $v(\emptyset)=0$ and $v(N)=1$. This fuzzy measure merely expresses the importance of each subset of points of view. The parentheses used for indexes represent a permutation on $N$ such that

$$
x_{(1)} \leqslant \cdots \leqslant x_{(n)},
$$

and $A_{(i)}$ represents the subset $\{(i), \ldots,(n)\}, A_{(n+1)}=\emptyset$ by convention.

If points of view cannot be considered as being independent, the importance of the combinations $S \subseteq N$, namely $v(S)$ has to be taken into account.

The Choquet integral presents standard properties for aggregation [10,18,11]: it is continuous, non decreasing, located between min and max.

The major advantage linked to the use of the Choquet integral derives from the large number of coefficients $\left(2^{n}-2\right)$ associated with a fuzzy measure. On the other hand, this flexibility can also be considered as a serious drawback, especially when assigning real values to the importance of all possible combinations.

Let us present an equivalent definition of the Choquet integral which will be used in the context of fuzzy partial evaluations. Let $v$ be a fuzzy measure on $N$. The Möbius transform of $v$ is a set function $m: 2^{N} \rightarrow \mathbb{R}$ defined by

$$
m(S)=\sum_{T \subseteq S}(-1)^{|S|-|T|} v(T)(S \subseteq N) .
$$

This transformation is invertible and thus constitutes an equivalent form of a fuzzy measure. $v$ can be recovered from $m$ by using

$$
v(S)=\sum_{T \subseteq S} m(T)(S \subseteq N) .
$$

This transformation can be used to redefine the Choquet integral without reordering the partial scores:

$$
\mathcal{C}_{m}(x)=\sum_{T \subseteq N} m(T) \bigwedge_{i \in T} x_{i}
$$

\footnotetext{
${ }^{2}$ The satisfaction of one point of view has almost the same effect as the satisfaction of both.

${ }^{3}$ The satisfaction of only one point of view is weak compared to the satisfaction of both points of view.

${ }^{4}$ Consider $x, y \in A$ for which the evaluations on $S \subseteq N$ are equal. The subset $N \backslash S$ is preferentially independent of $S$ if the preference of $x$ over $y$ is not influenced by their common part on $S$.
} 
A fuzzy measure is $k$-additive [8] if its Möbius transform $m$ satisfies $m(S)=0$ for $S$ such that $|S|>k$ and there exists at least one subset $S$ such that $|S|=k$ and $m(S) \neq \emptyset$.

Thus, $k$-additive fuzzy measures can be represented by at most $\sum_{i=1}^{k}\left(\begin{array}{c}n \\ i\end{array}\right)$ coefficients.

For a $k$-additive fuzzy measure,

$$
\mathcal{C}_{m}(x)=\sum_{\substack{T \subseteq N \\|T| \leqslant k}} m(T) \bigwedge_{i \in T} x_{i} .
$$

In order to be able to use fuzzy partial evaluations, we define the fuzzy extension of the Choquet integral in the following section.

\section{A fuzzy extension of the Choquet integral}

We can see that definition (1) of the Choquet integral in terms of a set function $m$ is a combination of functions which are continuous on $\mathbb{R} \times \mathbb{R}$, namely the addition $(+)$, the multiplication $(\cdot)$ and the minimum $(\wedge)$ functions.

By using the extension principle of Zadeh described in Section 2 one can extend these three functions to their fuzzy versions.

First of all, the extension $\widetilde{+}$ of the addition of two real numbers to two fuzzy numbers can be defined as

$$
\widetilde{x_{1}} \tilde{+} \widetilde{x_{2}}(y)=\sup _{a+b=y} \min \left[\widetilde{x_{1}}(a), \widetilde{x_{2}}(b)\right]
$$

The result is a fuzzy number according to [14]. In particular, the sum of two trapezoidal (resp. triangular) fuzzy numbers remains a trapezoidal (triangular) fuzzy number. Let us consider two trapezoidal fuzzy numbers $\widetilde{x}=\left(a_{x}, b_{x}, \alpha_{x}, \beta_{x}\right)$ and $\tilde{y}=\left(a_{y}, b_{y}, \alpha_{y}, \beta_{y}\right)$. According to the definition of the sum of two fuzzy numbers we have $\tilde{x} \tilde{+} \tilde{y}=\left(a_{x}+a_{y}, b_{x}+\right.$ $\left.b_{y}, \alpha_{x}+\alpha_{y}, \beta_{x}+\beta_{y}\right)$.

The multiplication, in our case, is a scalar multiplication of a fuzzy number by a crisp number $p \in \mathbb{R}$ (a coefficient of $m) . p \tilde{x} \tilde{x}$ is such that

$$
\left[p \tilde{x_{1}}\right]^{\lambda}=p\left[\widetilde{x_{1}}\right]^{\lambda}
$$

for any $\lambda \in[0,1]$. Again, the result of this scalar multiplication is a fuzzy number. In the particular case of a trapezoidal (triangular) fuzzy number, the output remains a trapezoidal (triangular) fuzzy number. For a trapezoidal number $\tilde{x}=$ $\left(a_{x}, b_{x}, \alpha_{x}, \beta_{x}\right)$ we have $p \tilde{x}=\left(p a_{x}, p b_{x}, p \alpha_{x}, p \beta_{x}\right)$.

Finally, the extension $\widetilde{\wedge}$ of the minimum of two crisp numbers becomes

$$
\widetilde{\bigwedge}\left(\tilde{x_{1}}, \tilde{x_{2}}\right)(y)=\sup _{\min (a, b)=y} \min \left[\tilde{x_{1}}(a), \tilde{x_{2}}(b)\right]
$$

Again the result is a fuzzy number. But in the special case of trapezoidal or triangular fuzzy numbers, the result no longer remains a trapezoidal or triangular fuzzy number. The side functions become piecewise linear functions. Fig. 1 represents the minimum (bold dashes) of two fuzzy numbers.

Now that we have presented the extensions of the operators needed to use the Choquet integral as an aggregation operator for fuzzy numbers, we define the fuzzy extension of the Choquet integral as

$$
\widetilde{\mathcal{C}_{m}}(x)=\widetilde{\sum_{T \subseteq N}} m(T) \sim \widetilde{\bigwedge_{i \in T}} \tilde{x}_{i},
$$

where $m$ is the set function which is obtained by a Möbius transform of the fuzzy measure $v$.

The Choquet integral of a vector of fuzzy partial evaluations is a fuzzy number. In case of partial evaluations which are trapezoidal or triangular fuzzy numbers, the resulting fuzzy number has piecewise linear side functions. 


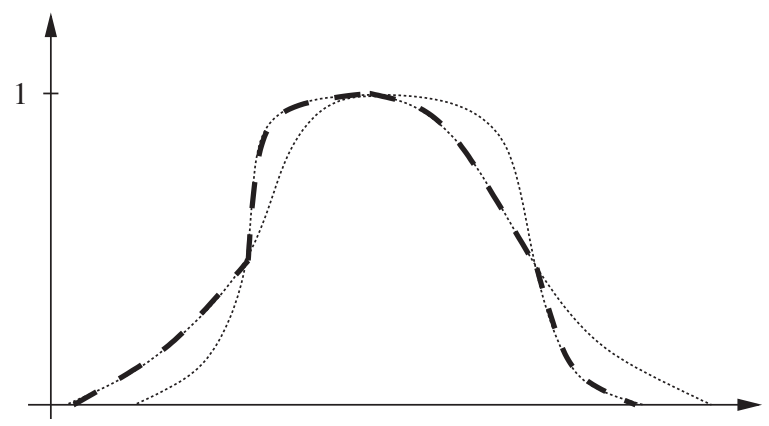

Fig. 1. Minimum of two fuzzy numbers (bold dashes).
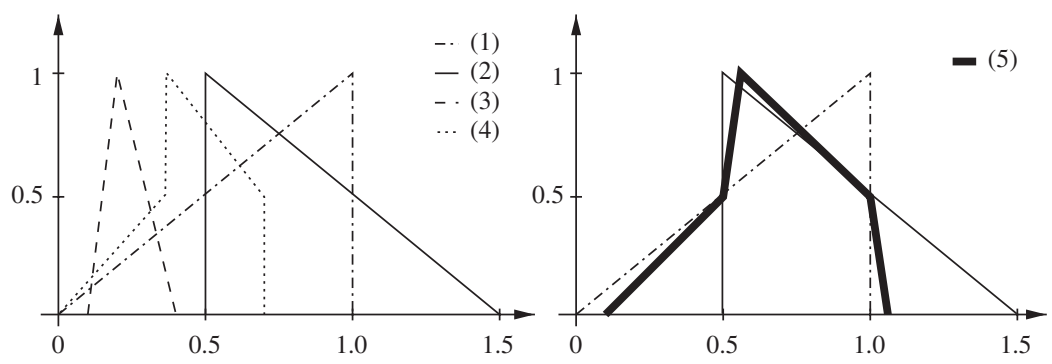

Fig. 2. Example 1: towards the aggregation.

It is obvious that this extension remains valid in the case of a $k$-additive fuzzy measure. The definition can then be written

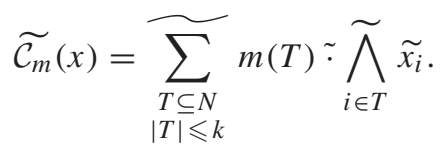

Let us consider a short example to show how the Choquet integral is calculated in case of triangular partial evaluations. This example is taken from [19] (Example 5.1).

Example 1. Let $N=\{1,2\}, v(\{1\})=0.1, v(\{2\})=0.2, v(\{1,2\})=1$. In terms of the set function $m$ (the Möbius transform of $v$ ) defined previously this problem can be rewritten as $m(\{1\})=0.1, m(\{2\})=0.2, m(\{1,2\})=0.7$. The partial evaluations are represented by the triangular fuzzy numbers $\widetilde{x_{1}}=(1,1,0)$ and $\widetilde{x_{2}}=(0.5,0,1)$. The Choquet integral then becomes:

$$
\begin{aligned}
& \widetilde{\mathcal{C}_{m}}\left(\left(\widetilde{x_{1}}, \widetilde{x_{2}}\right)\right)=m(\{1\}) \sim \widetilde{x_{1}} \tilde{+} m(\{2\}) \sim \widetilde{x_{2}} \tilde{+} m(\{1,2\}) \sim \widetilde{\Lambda}\left(\widetilde{x_{1}}, \widetilde{x_{2}}\right) \\
& =(0.2,0.1,0.2) \tilde{+} 0.7 \sim \widetilde{\Lambda}\left(\tilde{x_{1}}, \tilde{x_{2}}\right) \\
& :=\tilde{s} \tilde{+} \tilde{s}^{\prime},
\end{aligned}
$$

where $\tilde{s}=(0.2,0.1,0.2)$ and $\tilde{s^{\prime}}=0.7 \sim \tilde{\wedge}\left(\widetilde{x_{1}}, \widetilde{x_{2}}\right)$. Fig. 2 shows a few steps of these calculations. (1) and (2) stand respectively for $\tilde{x_{1}}$ and $\widetilde{x_{2}}$. (3) and (4) represent $\widetilde{s}$ and $\widetilde{s^{\prime}}$. Finally, (5) shows the aggregated value $\widetilde{\mathcal{C}_{m}}\left(\left(\widetilde{x_{1}}, \widetilde{x_{2}}\right)\right)$.

In [19] a fuzzy extension of the Choquet integral is presented, based on the classical representation of the Choquet integral by means of a fuzzy measure. A difficulty in that case is the ordering of the fuzzy numbers which is not needed in our approach and is problematic in the case of non linear fuzzy numbers. A numerical method is developed to estimate the value of the Choquet integral using trapezoidal fuzzy numbers. The authors of [2] present an interval-based Choquet integral to derive preferences on multicriteria alternatives. 


\section{Ranking the alternatives and determining a best choice}

Let us suppose that the partial evaluations of the elements of $A$ have been aggregated into a fuzzy number for each alternative. We will see later in Section 7 how to determine the values of the coefficients of the fuzzy measure $v$ (or equivalently of its Möbius transform $m$ ). The global evaluations obtained by the aggregation will allow us to determine a ranking or a best choice on the set $A$ which can be proposed to the decision maker.

To build such a ranking, we suggest two possibilities: a complete preorder and a complete interval order. By means of a fuzzy preference digraph, we show how to obtain a solution for the best choice problem. These three proposals are described in the following three subsections.

To obtain the rankings we use the concept of possibilistic mean. Recall what has been said in Section 2 and consider the membership function of a fuzzy number $\tilde{x}$ as a possibility distribution. We then define the upper possibilistic mean of $\tilde{x}$ by

$$
\mathcal{M}^{+}(\tilde{x}):=\int_{0}^{1} a_{M}(\lambda) \mathrm{d} \lambda=\int_{0}^{1} a_{M}(\lambda) \operatorname{dPos}\left(\tilde{x} \geqslant a_{M}(\lambda)\right)
$$

and the lower possibilistic mean of $\tilde{x}$ by

$$
\mathcal{M}^{-}(\tilde{x}):=\int_{0}^{1} a_{m}(\lambda) \mathrm{d} \lambda=\int_{0}^{1} a_{m}(\lambda) \mathrm{dPos}\left(\tilde{x} \leqslant a_{m}(\lambda)\right) .
$$

The possibilistc mean of the fuzzy number $\tilde{x}$ is then defined by

$$
\mathcal{M}(\tilde{x}):=\frac{1}{2}\left[\mathcal{M}^{+}(\tilde{x})+\mathcal{M}^{-}(\tilde{x})\right]
$$

Fig. 3 gives a representation of the upper possibilistic mean in terms of a real number and a surface.

If we consider two fuzzy numbers $\tilde{x}$ and $\tilde{y}$ we can define the upper dominance $\stackrel{+}{\geqslant}$ of $\tilde{x}$ over $\tilde{y}$ by

$$
\tilde{x} \geqslant \tilde{y} \Longleftrightarrow \mathcal{M}^{+}(\tilde{x})-\mathcal{M}^{-}(\tilde{y}) \geqslant 0 .
$$

Similarly one can define the lower dominance $\stackrel{-}{\geqslant}$.

\subsection{Ranking by a complete preorder}

The definition of the possibilistic mean can be applied to the global evaluation of an alternative $x$ of $A$ by means of the fuzzy extension of the Choquet integral $\widetilde{\mathcal{C}_{m}}(x)$. Let $x$ and $y$ be two alternatives of $A$. A complete preorder is then defined by the relation $\succeq$ (is not worse than)

$$
x \succeq y \Longleftrightarrow \mathcal{M}\left(\widetilde{\mathcal{C}_{m}}(x)\right) \geqslant \mathcal{M}\left(\widetilde{\mathcal{C}_{m}}(y)\right) .
$$

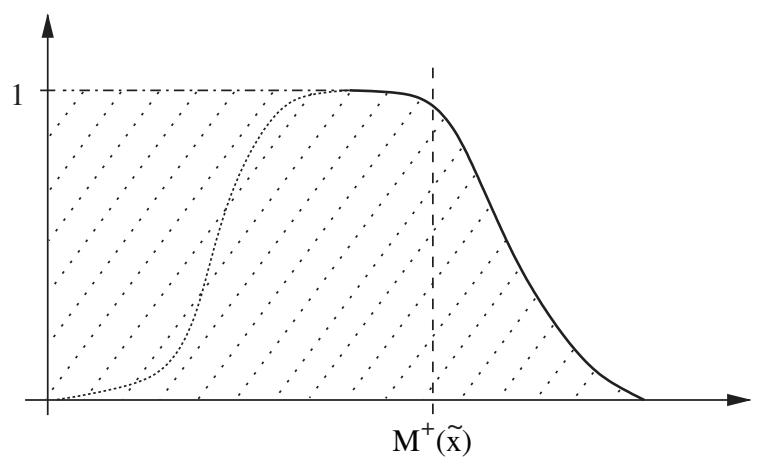

Fig. 3. Representations of $\mathcal{M}^{+}(\tilde{x})$ : a number and a surface. 


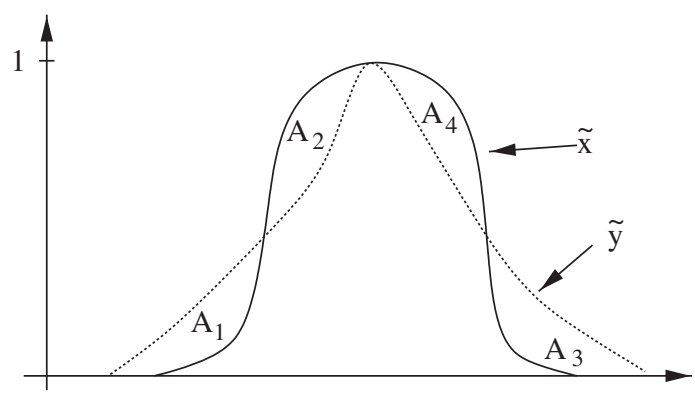

Fig. 4. Comparing $\tilde{x}$ to $\tilde{y}$.

It is possible to give a geometric interpretation to this relation. Let us consider a situation with two fuzzy numbers $\tilde{x}$ and $\widetilde{y}$ which represent the global evaluation of two alternatives $x$ and $y$ of $A$. These two fuzzy numbers define four areas $A_{1}, \ldots, A_{4}$ as shown on Fig. 4. We see that $A_{4}-A_{3}=\mathcal{M}^{+}(\widetilde{x})-\mathcal{M}^{+}(\widetilde{y})$ (upper dominance) and $A_{1}-A_{2}=$ $\mathcal{M}^{-}(\tilde{x})-\mathcal{M}^{-}(\tilde{y})$ (lower dominance). Definition 2 can then be rewritten as

$$
x \succeq y \Longleftrightarrow A_{4}+A_{1} \geqslant A_{3}+A_{2} .
$$

This complete preorder proposal corresponds to the area compensation method of Fortemps and Roubens [7].

\subsection{Ranking by a complete interval order}

This approach is based on the comparison of the intervals $\left[\mathcal{M}^{-}\left(\widetilde{\mathcal{C}_{m}}(x)\right), \mathcal{M}^{+}\left(\widetilde{\mathcal{C}_{m}}(x)\right)\right]$, for each $x$ in $A$. Let $I$ be a symmetrical relation, and $P$ be an antisymmetrical relation on $A \times A$ such that

$$
\left\{\begin{array}{l}
x P y \quad \Longleftrightarrow \mathcal{M}^{-}\left(\widetilde{\mathcal{C}_{m}}(x)\right)>\mathcal{M}^{+}\left(\widetilde{\mathcal{C}_{m}}(y)\right), \\
y P x \quad \Longleftrightarrow \quad \mathcal{M}^{-}\left(\widetilde{\mathcal{C}_{m}}(y)\right)>\mathcal{M}^{+}\left(\widetilde{\mathcal{C}_{m}}(x)\right), \\
x I y \text { else. }
\end{array}\right.
$$

The relation $(P, I)$ is an interval order. It is now possible to build a ranking on the alternatives of $A$. The particularity of such an interval order is that the associated indifference relation $I$ is not transitive. For three alternatives $x, y$ and $z$ of $A$ it is possible to have the situation where $x I y$ and $y I z$, but $x P z$.

\subsection{Choosing in a fuzzy preference digraph}

This proposal extracts a subset of alternatives which can be considered as the "best" ones. This subset is called a best choice recommendation and each alternative which does not belong to the choice should be rejected. It is based on a degree of credibility of the preference of one alternative on another one. One can define this credibility as the possibility $\Pi$ that an alternative $x$ is not worse than $y$ (let's write $x \succeq y$ ) in the following way:

$$
\Pi(x \succeq y)=\sup _{a \geqslant b}[\min (\widetilde{x}(a), \tilde{y}(b))] .
$$

Fig. 5 illustrates the meaning of this degree of credibility of the preference of $x$ over $y(x, y \in A)$. $h$ represents the height at the intersection of the two fuzzy numbers $\tilde{x}$ and $\tilde{y}$ representing $x$ and $y$. We have $\Pi(x \succeq y)=h$ and $\Pi(y \succeq x)=1$.

Roubens and Vincke [17] have shown that the credibility $\Pi$ as defined in (3) is a fuzzy interval order (a reflexive, complete $^{5}$ and Ferrers ${ }^{6}$ valued relation) and that it is min-max-transitive. If these credibilities are computed for each pair of alternatives of $A$, it is possible to represent the problem as a valued digraph where the nodes represent the alternatives and the arcs the valued relation $\Pi$. This graph is called a fuzzy possibility digraph.

\footnotetext{
$5 x \Pi y$ or $y \Pi x$ for each $x \neq y$.

${ }^{6} w \Pi x, y \Pi z \Rightarrow w \Pi z$ or $z \Pi x$.
} 


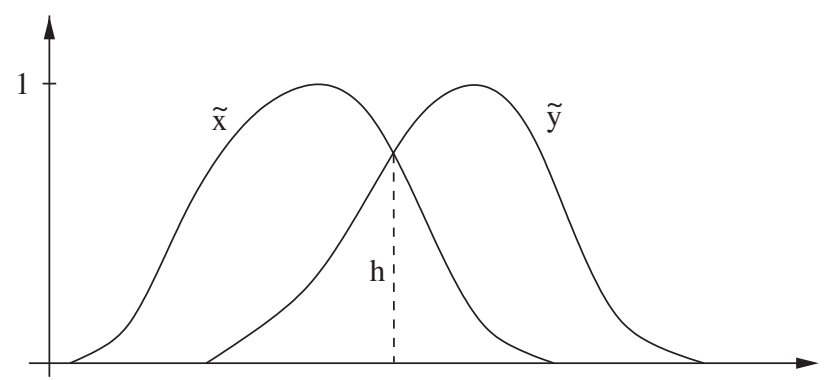

Fig. 5. Degree of credibility of the preference of $x$ over $y$.

Starting from this strongly complete relation $\Pi$, one can define a preference relation by

$$
P(x, y)=1-\Pi(y \succeq x) \quad \forall(x, y) \in A^{2}[6] .
$$

The associated digraph is called a fuzzy preference digraph. The goal is now to extract some useful information from this digraph, in order to determine a best choice among the alternatives of $A$. Let us first recall the concept of the score of non-domination of an alternative $x$ of $A$ [15]

$$
N D(x)=1-\max _{y \neq x} P(y, x) .
$$

The core $Y_{0}$ of $A$ is a subset $Y_{0} \subset A$ such that its elements all have a score of non-domination of 1 . In other words, $Y_{0}=\{x \in A \mid N D(x)=1\}$. It has been shown in [17] that the core of a fuzzy preference digraph is non-empty.

$Y_{0}$ gives a solution to the best choice problem (the subset of best actions among $\mathrm{A}$ ).

\subsection{On the calculation of the possibilistic means in practise}

In real-life multiple criteria decision support problems, one can suppose that the partial evaluations of the alternatives are given by trapezoidal or triangular fuzzy numbers. Besides the objective can be to build the simplest possible model, which means in our framework to use a $k$-additive Choquet integral with $k$ as low as possible.

Let us, therefore, suppose that the partial evaluations of an alternative $x$ are trapezoidal fuzzy numbers $\tilde{x}_{i}=$ $\left(a_{i}, b_{i}, \alpha_{i}, \beta_{i}\right)(i \in N)$ and that we restrict to a 2-additive Choquet integral. The aggregation can then be written

$$
\widetilde{\mathcal{C}_{m}}(x)=\widetilde{\sum_{i=1}^{n}} m(\{i\}) \tilde{x_{i}} \tilde{+} \widetilde{\sum_{\{i, j\} \subseteq N}^{n}} m(\{i, j\}) \sim \widetilde{\min }\left(\tilde{x_{i}}, \tilde{x_{j}}\right) .
$$

The minimum of two trapezoidal fuzzy numbers can be summarised by eight parameters $\left(a, b, \lambda^{-}, \alpha^{\prime}, \alpha^{\prime \prime}, \lambda^{+}, \beta^{\prime}, \beta^{\prime \prime}\right)$ representing two upper piecewise linear shape functions passing through the following points: $\left(a-\alpha^{\prime \prime}, 0\right),\left(a-\alpha^{\prime}, \lambda^{-}\right)$, $(a, 1),(b, 1),\left(b+\beta^{\prime}, \lambda^{+}\right),\left(b+\beta^{\prime \prime}, 0\right)$. Fig. 6 represents these eight parameters. Let $\tilde{m}$ be the minimum of two trapezoidal fuzzy numbers. One can easily obtain that

$$
\mathcal{M}^{-}(\tilde{m})=a-\frac{\alpha^{\prime}+\lambda^{-} \alpha^{\prime \prime}}{2}
$$

and

$$
\mathcal{M}^{+}(\tilde{m})=b+\frac{\beta^{\prime}+\lambda^{+} \beta^{\prime \prime}}{2} .
$$

Furthermore, one can see that for any fuzzy numbers $\tilde{x}$ and $\tilde{y}$ and any real number $p, \mathcal{M}^{ \pm}(p \tilde{x})=p \mathcal{M}^{ \pm}(\tilde{x})$ and $\mathcal{M}^{ \pm}(\tilde{x} \tilde{+} \tilde{y})=\mathcal{M}^{ \pm}(\tilde{x})+\mathcal{M}^{ \pm}(\tilde{y})^{7}$.

\footnotetext{
${ }^{7} \mathcal{M}^{ \pm}$is an abbreviation to avoid the writing of two similar formulae for $\mathcal{M}^{+}$and for $\mathcal{M}^{-}$.
} 


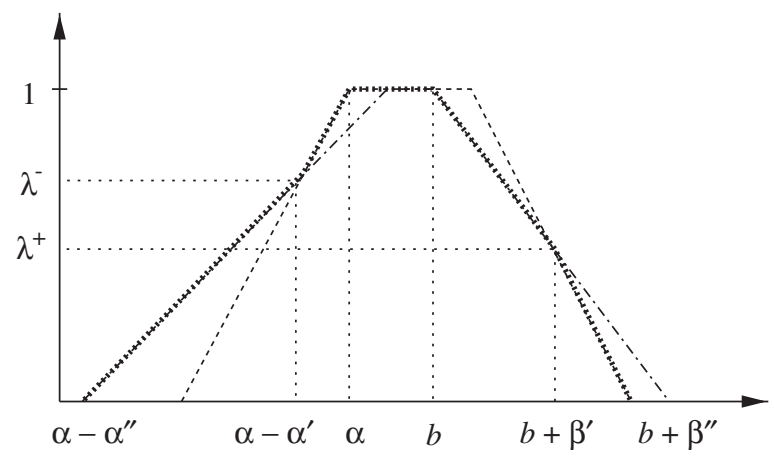

Fig. 6. The minimum of two trapezoidal fuzzy numbers.

For any alternative $x \in A$ we therefore have

$$
\mathcal{M}^{ \pm}\left[\widetilde{\mathcal{C}_{m}}(x)\right]=\sum_{i=1}^{n} m(\{i\}) \mathcal{M}^{ \pm}\left(\tilde{x_{i}}\right)+\sum_{\substack{i, j=1 \\ i \neq j}}^{n} m(\{i, j\}) \mathcal{M}^{ \pm}\left(\min \left(\tilde{x_{i}}, \tilde{x_{j}}\right)\right) .
$$

This shows that in this particular case where the partial evaluations are trapezoidal fuzzy numbers and where we restrict to a 2-additive fuzzy number, the possibilistic mean of the global evaluations of the alternatives can be very easily calculated.

The following section shows how to determine the coefficients of the set function $m$ used in the definition of the fuzzy extension of the Choquet integral.

\section{Assessment of the coefficients of $m$}

The method described in Section 6 presents how to build two possible rankings and a best choice recommendation on alternatives which have fuzzy partial evaluations on a set of criteria. These evaluations are aggregated by means of a fuzzy extension of the Choquet integral presented in Section 5. One important point concerns the assessment of the coefficients of the set function $m$ which is the Möbius transform of the fuzzy measure $v$ (see Section 4).

Even if one restricts the model to a 2-additive Choquet integral, the number of coefficients which have to be determined for the aggregation is $\sum_{i=1}^{2}\left(\begin{array}{c}n \\ i\end{array}\right)=n(n+1) / 2$. It seems obvious that neither the coefficients of the fuzzy measure $v$, nor those of its Möbius transform $m$ have a clear meaning to the decision maker. Therefore the large number of coefficients linked to an unclear semantics of $v$ or $m$ lead us to think that an alternative possibility has to be explored.

In [13] we have explored the sorting of alternatives by means of a Choquet integral in a universe of crisp partial evaluations. The idea is to ask the decision maker to provide information on a set of prototypes. A prototype is an alternative which is well known to the decision maker and which belongs to his domain of expertise.

A decisive hypothesis has to be done here. As a prototype is a well-known alternative for the decision maker, we suppose that it is also well-defined, and has therefore crisp partial evaluations. We are aware that this assumption is very strong and may be a weakness of our proposal. We nevertheless think that it is reasonable to suppose that the decision maker, as an expert of a particular knowledge field, can provide a few of these well-defined alternatives.

Let us suppose that the decision maker has provided a set $P$ of such prototypes. We then ask him to determine a total order on the elements of $P$. Once again, as he is an expert of the knowledge field we are working on, he should be able to provide such an order.

The next step is to determine the coefficients of the set function $m$ used in the definition of the Choquet integral. As we have no guarantee that the total order provided by the decision maker is compatible with a discrimant function of the Choquet integral type, ${ }^{8}$ the objective is to determine a set function $m$ which will provide a satisfactory ranking on

\footnotetext{
${ }^{8}$ The partial order may violate the triple cancellation axiom $[10,18]$ for example.
} 
the prototypes. Therefore, the following procedure tends to minimise the gap between the ranking given by the decision maker and the one resulting from the aggregation. Intuitively, for a given alternative $x \in P$, its Choquet integral $\mathcal{C}_{m}(x)$ should be as close as possible to an unknown global evaluation $y(x)$, which respects the total order imposed by the decision maker on the prototypes.

The coefficients of the set function $m$ are determined by solving the following quadratic program:

$$
\min \sum_{x \in P}\left[\mathcal{C}_{m}(x)-y(x)\right]^{2},
$$

where the unknowns are:

- the coefficients of the set function $m$;

- some global evaluations $y(x)$ for each $x \in P$.

In order to ensure boundary and monotonicity conditions imposed on the fuzzy measure $v$, its Möbius transform $m$ must satisfy [3]:

$$
\left\{\begin{array}{l}
m(\emptyset)=0, \quad \sum_{T \subseteq N} m(T)=1, \\
\sum_{T: i \in T \subseteq S} m(T) \geqslant 0, \quad \forall S \subseteq N, \quad \forall i \in S .
\end{array}\right.
$$

The global evaluations $y(x)$ must verify the ranking imposed by the decision maker. In other words, for every ordered pair $\left(x, x^{\prime}\right) \in P \times P$, the condition $y(x)-y\left(x^{\prime}\right) \geqslant \varepsilon, \varepsilon>0$ must be satisfied ${ }^{9}$.

The solution of the quadratic program gives the coefficients of the set function $m$ and the global evaluations $y(x), \forall x \in$ $A$. If the objective function equals 0 , then for each alternative, its global evaluation corresponds to its aggregated evaluation by a Choquet integral. In that case, the ranking obtained by ordering the alternatives according to their aggregated evaluations is consistant with the ranking on the prototypes. If the objective function is strictly positive, then it was not possible to determine the coefficients of the set function $m$ in order to respect the ranking on the prototypes. In the ranking obtained by ordering the alternatives according to their aggregated evaluations, certain pairs of alternatives may be reversed compared to the ranking imposed on the prototypes.

It is possible to measure the adequacy of both rankings. The symmetric difference distance $\delta$ defined as

$$
\delta(R, S)=\mid\left\{(a, b) \in A^{2}:[x R y \text { and } \neg(x S y)] \text { or }[\neg(x R y) \text { and }(x S y)]\right\} \mid,
$$

where $R$ and $S$ are two binary relations on $A$, measures the number of disagreements between $R$ and $S$. Barthélemy [1] shows that $\delta$ satisfies a number of naturally desirable properties. In our case, we can measure the symmetric difference distance between the ranking imposed on the prototypes, and the one obtained by the use of a Choquet integral. By using $\delta$ it is now quite easy to measure the adequacy of both rankings.

If the decision maker is satisfied with the model built on the prototypes (let us suppose that the $\delta$ distance is small), the next step is to determine the global evaluations of the alternatives of $A$ which have fuzzy partial evaluations. The coefficients of the set function $m$, determined by the procedure we just described, are used to aggregate the fuzzy partial evaluations by means of the extension of the Choquet integral presented in Section 5.

\section{Concluding remarks}

In this paper, we have presented an extension of the Choquet integral to fuzzy partial evaluations in the case of multiple criteria decision support. This extension is very simple as we use the representation of the Choquet integral by means of the Möbius transform. We have presented two rankings and a best choice recommendation on the alternatives and we have shown how it is possible to determine the coefficients of the Choquet integral.

\footnotetext{
${ }^{9}$ In practise, one of the main problems is to fix the value of $\varepsilon$. We think that it has to be chosen in ]0,1/p[ where $p$ is the number of prototypes considered by the decision maker.
} 


\section{References}

[1] J.-P. Barthélemy, Caractérisations axiomatiques de la distance de la différence symétrique entre des relations binaires, Math. Sci. Humaines 67 (1979) 85-113.

[2] M. Ceberio, F. Modave, An interval-valued 2-additive Choquet integral for multicriteria decision making, in: Proc. 10th Conference IPMU, July 2004, Perugia, Italy, 2004, pp. 1567-1574.

[3] A. Chateauneuf, J.-Y. Jaffray, Some characterizations of lower probabilities and other monotone capacities through the use of Möbius inversion, Math. Social Sci. 17 (3) (1989) 263-283.

[4] G. Choquet, Theory of capacities, Ann. Inst. Fourier 5 (1953) 131-295.

[5] D. Dubois, H. Prade, Fuzzy Sets and Systems: Theory and Application, Academic Press, New York, 1980.

[6] J. Fodor, M. Roubens, Fuzzy Preference Modelling and Multicriteria Decision Support, Theory and Decision Library, Series D: System Theory, Knowledge Engineering and Problem Solving, Kluwer Academic Publishers, Dordrecht, Boston, London, 1994.

[7] P. Fortemps, M. Roubens, Ranking and defuzzification methods based on area compensation, Fuzzy Sets and Systems 82 (1996) 319-330.

[8] M. Grabisch, $k$-order additive discrete fuzzy measure and their representation, Fuzzy Sets and Systems 92 (1997) 131-295.

[9] M. Grabisch, M. Roubens, Fuzzy measures and integrals, in: Grabisch et al. (Eds.), Fuzzy Sets and Their Applications, Physica-Verlag, Heidelberg, 2000.

[10] J.-L. Marichal, An axiomatic approach of the discrete Choquet integral as a tool to aggregate interacting criteria, IEEE Trans. Fuzzy Systems 8 (2000) 800-807.

[11] J.-L. Marichal, Aggregation of interacting criteria by means of the discrete Choquet integral, in: T. Calvo, G. Mayor, R. Mesiar (Eds.), Aggregation Operators: New Trends and Applications, Studies in Fuzziness and Soft Computing, vol. 97, Physica-Verlag, Heidelberg, 2002, pp. 224-244.

[12] J.-L. Marichal, P. Meyer, M. Roubens, Sorting multiattribute alternatives: The TOMASO method, Internat. J. Comput. Oper. Res. 32 (2005) 861-877.

[13] P. Meyer, M. Roubens, Choice, ranking and sorting in fuzzy multiple criteria decision aid, in: J. Figueira, S. Greco, M. Ehrgott (Eds.), Multiple Criteria Decision Analysis: State of the Art Surveys, Kluwer Academic Publishers, Dordrecht, 2005, pp. 471-506.

[14] H.T. Nguyen, A note on the extension principle for fuzzy sets, J. Math. Anal. Appl. 64 (1978) 369-380.

[15] S.A. Orlovski, Decision-making with a fuzzy preference relation, Fuzzy Sets and Systems 1 (1978) 155-167.

[16] F.S. Roberts, Measurement Theory, Addison-Wesley, Reading, MA, 1979.

[17] M. Roubens, P. Vincke, Fuzzy possibility graphs and their application to ranking fuzzy numbers, in: M. Roubens, J. Kacprzyk (Eds.), NonConventional Preference Relations in Decision Making, Springer, Berlin, 1988, pp. 119-128.

[18] P. Wakker, Additive Representations of Preferences: A New Foundation of Decision Analysis, Kluwer Academic Publishers, Dordrecht, Boston, London, 1989.

[19] R. Yang, Z. Wang, P.-A. Heng, K.-S. Leung, Fuzzy numbers and fuzzification of the Choquet integral, Fuzzy Sets and Systems 153 (2005) 95-113.

[20] L.A. Zadeh, Fuzzy sets, Inform. Control 8 (1965) 338-353.

[21] L.A. Zadeh, Fuzzy sets as a basis for a theory of possibility, Fuzzy Sets and Systems 1 (1978) 3-28. 